\title{
New nonlinear coherent states and some of their nonclassical properties
}

\author{
B.Roy.* and P. Roy ${ }^{\dagger}$ \\ Physics \& Applied Mathematics Unit \\ Indian Statistical Institute \\ Calcutta 700035 \\ India
}

October 27, 2018

\begin{abstract}
We construct a displacement operator type nonlinear coherent state and examine some of its properties. In particular it is shown that this nonlinear coherent state exhibits nonclassical properties like squeezing and sub-Poissonian behaviour.
\end{abstract}

PACS No: 42.50Dv, 42.50p

*E-mail : barnana@isical.ac.in

${ }^{\dagger}$ E-mail : pinaki@isical.ac.in 


\section{Introduction}

The importance of coherent states of various Lie algebras in different branches of physics(in particular quantum optics) hardly needs to be emphasized. For example the standard coherent state of the harmonic oscillator corresponds to the Heisenberg-Weyl algebra. Similarly coherent states corresponding to Lie algebras like $\mathrm{Su}(1,1), \mathrm{Su}(2)$ can also be constructed and they have also found numerous applications in quantum optics [1, 2]. In this connection it may be mentioned that coherent states are usually constructed using any of the following three procedures : (1) Displacement operator technique (2) Annihilation operator eigenstates (3) Minimum uncertainty states. However,these three approaches are generally nonequivalent and only in the case of standard harmonic oscillator coherent states obtained using any of the three approaches are equivalent.

On the other hand nonlinear coherent states [3] or the f-coherent states [4] are coherent states corresponding to nonlinear algebras rather than Lie algebras. Nonlinear algebras are distinct from Lie algebras and have recently been used to analyse a number of quantum mechanical systems [5, 6, 7, 8]. However nonlinear coherent states are not merely mathematical objects but they are useful too. Recently it has been shown that nonlinear coherent states are useful in the description of the motion of a trapped ion and various nonclassical properties of such states have also been studied [3]. We note that in refs [3] and [4] nonlinear coherent states have been defined as the right eigenstate of a generalised annihilation operator $A$ (which emerges from the Hamiltonian describing the dynamics). This is because in the case of nonlinear algebras the commutator $\left[A, A^{\dagger}\right]$ is not a constant or a linear function of the generators of the algebra but nonlinear in the generators. As a consequence it is difficult to obtain an explicit form of nonlinear coherent state constructed via the displacement operator technique.

In the present paper our aim is to construct nonlinear coherent states using an operator which is similar to the displacement operator. This approach has been used previously to construct nonlinear coherent states in the context of isospectral Hamiltonians [9, 10] and deformed algebras [11, 12]. Subsequently we shall examine various nonclassical properties

like quadrature as well as amplitude squared squeezing,sub-Poissonian behaviour etc. of the nonlinear coherent states so obtained. The organisation of the paper is as follows: in section 2 we shall describe the construction of nonlinear coherent states; in section 3 we shall shall study nonclassical properties of the nonlinear coherent state; finally section 4 
is devoted to a conclusion.

\section{Construction of nonlinear coherent states using a displacement type operator}

To begin with we note that the generalised annihilation (creation) operator associated with nonlinear coherent states are given by

$$
A=a f(N), \quad A^{\dagger}=f(N) a^{\dagger}, \quad N=a^{\dagger} a
$$

where $f(x)$ is a reasonably well behaved real function and $a^{\dagger}(a)$ is the harmonic oscillator creation(annihilation) operator. It can be easily verified that $A^{\dagger}, A$ and $N$ satisfy the following nonlinear algebra:

$$
[N, A]=-A, \quad\left[N, A^{\dagger}\right]=A^{\dagger} \quad, \quad\left[A, A^{\dagger}\right]=(N+1) f^{2}(N+1)-N f^{2}(N)
$$

Clearly the nature of the nonlinear algebra depends on the choice of the nonlinearity

function $f(N)$. If however $f(N)=1$ then the nonlinear algebra in (2) reduces to the Heisenberg algebra.

Nonlinear coherent states $\mid \alpha>$ are then defined as right eigenstates of the generalised annihilation operator $A$ [3, 幽:

$$
A|\alpha>=\alpha| \alpha>
$$

where $\alpha$ is an arbitrary complex number. From (3) we can now obtain an explicit form of the nonlinear coherent state in a number state representation:

$$
\left|\alpha>=C \sum_{n=0}^{\infty} \alpha^{n} d_{n}\right| n>
$$

where the coefficients $d_{n}$ 's are given by

$$
d_{0}=1, d_{n}=[\sqrt{n} ! f(n) !]^{-1}, f(n) ! \equiv f(1) \ldots f(n)
$$

and the normalisation constant $\mathrm{C}$ can be obtained from the condition $\langle\alpha \mid \alpha\rangle=1$ and is given by

$$
C^{2}=\left[\sum_{n=0}^{\infty} d_{n}^{2}|\alpha|^{2 n}\right]^{-1}
$$


We now turn to the construction of a new type of nonlinear coherent state. From the relation (2) we find that the r.h.s of the commutator $\left[A, A^{\dagger}\right]$ is neither a constant nor linear in the generators but is a nonlinear function of the generator $N$. As a result $\mathrm{BCH}$ disentangling theorem [13] can not be applied and one can not use the displacement operator $\exp \left(\alpha A-\alpha^{*} A^{\dagger}\right)$ to construct coherent states (see ref 14 for some recent results concerning the applicability of $\mathrm{BCH}$ disentangling theorem to nonlinear algebras).

We now proceed to determine an operator $B^{\dagger}$ which is conjugate of the operator $A$. In other words $A$ and $B^{\dagger}$ satisfy the commutation relation

$$
\left[A, B^{\dagger}\right]=1
$$

while their hermitian conjugates $A^{\dagger}$ and $B$ satisfy the dual algebra

$$
\left[B, A^{\dagger}\right]=1
$$

Then from (四),(7) and (8) it follows that

$$
B=a \frac{1}{f(N)}, \quad B^{\dagger}=\frac{1}{f(N)} a^{\dagger}
$$

Let us now consider the following operators ( $\beta$ being an arbitrary complex number):

$$
\begin{aligned}
& D(\beta)=\exp \left(\beta A^{\dagger}-\beta^{*} B\right) \\
& D_{1}(\beta)=\exp \left(\beta B^{\dagger}-\beta^{*} A\right)
\end{aligned}
$$

and note that for any two operators $X$ and $Y$ satisfying the relation $[X, Y]=1$ the $\mathrm{BCH}$ theorem [13] yields

$$
\exp \left(\beta X-\beta^{*} Y\right)=\exp \left(-\frac{\beta \beta^{*}}{2}\right) \exp (\beta X) \exp \left(-\beta^{*} Y\right)
$$

We now define nonlinear coherent states corresponding to the algebra (7) as $D(\alpha) \mid 0>$ while those corresponding to the dual algebra (8) as $D_{1}(\beta) \mid 0>$. Let us first consider the second case. Applying $D_{1}(\beta)$ on $\mid 0>$ we obtain on using (11)

$$
\left|\beta>_{1}=D_{1}(\beta)\right| 0>=c_{1} \sum_{n=0}^{\infty} \frac{\beta^{n}}{\sqrt{n} ! f(n) !} \mid n>
$$

where $c_{1}$ is a normalisation constant. Comparing (41) and (12) it is seen that the coherent state $\mid \beta>_{1}$ is the same as the nonlinear coherent state $\mid \alpha>$. Thus the nonlinear coherent 
state $\mid \alpha>$ can not only be obtained as an annihilation operator eigenstate but it can also be obtained by the application of a displacement type operator.

Let us now turn to the first possibility. Applying the operator $D(\beta)$ on $\mid 0>$ we get

$$
|\beta>=D(\beta)| 0>=c \sum_{n=0}^{\infty} \frac{\beta^{n} f(n) !}{\sqrt{n} !} \mid n>
$$

where as before $c$ is a normalisation constant and we have used (11) to obtain (13). The normalisation constant $c$ can be determined from the condition $\langle\beta \mid \beta\rangle=1$ and we get

$$
c^{2}=\left[\sum_{n=0}^{\infty} \frac{\left(\beta^{*} \beta\right)^{n}(f(n) !)^{2}}{n !}\right]^{-1}
$$

where $f(0) ! \equiv 0$. The superposition state obtained in (13) is the new nonlinear coherent state and this is distinct from the nonlinear coherent state defined in (4)(provided of course we use the same nonlinearity function $f(n)$ in both the cases). In the next section we shall study various properties of the nonlinear coherent state (13).

\section{Non classical properties of nonlinear coherent state $\mid \beta>$}

In this section we shall examine squeezing and antibunching properties of the new nonlinear coherent state $\mid \beta>$. However before we proceed any further it is necessary to specify the nonlinearity function $f(n)$. It is clear that for every choice of $f(n)$ we shall have a different nonlinear coherent states. In the present case we choose the following nonlinearity function which is useful in the description of the motion of a trapped ion [3]:

$$
f(n)=L_{n}^{1}\left(\eta^{2}\right)\left[(n+1) L_{n}^{0}\left(\eta^{2}\right)\right]^{-1}
$$

where $L_{n}^{m}(x)$ are generalised Laguerre polynomials and $\eta$ is known as the Lamb-Dicke parameter. Clearly $f(n)=1$ when $\eta=0$ and in this case nonlinear coherent states become the standard coherent states. However when $\eta \neq 0$ nonlinearity starts developing with the degree of nonlinearity depending on the magnitude of $\eta[3$, 15].

\subsection{Quadrature Squeezing}

Here we shall study quadrature squeezing of the new nonlinear coherent state $\mid \beta>$. In order to do so let us consider the following hermitian quadrature operators:

$$
X_{1}=\frac{\left(a+a^{\dagger}\right)}{2} \quad, \quad Y_{1}=\frac{\left(a-a^{\dagger}\right)}{2 i}
$$


Then $X_{1}$ and $Y_{1}$ satisfy the following uncertainty relation:

$$
<\Delta X_{1}^{2}>\quad<\Delta Y_{1}^{2}>\geq \frac{1}{16}
$$

where $\left\langle\Delta X^{2}\right\rangle=\left\langle X^{2}>-<X\right\rangle^{2}$. From (17) it follows that a state is squeezed if any of the following conditions hold:

$$
\begin{aligned}
& \left.<\Delta X_{1}^{2}\right\rangle<\frac{1}{4} \\
& \text { or } \\
& \left\langle\Delta Y_{1}^{2}\right\rangle \quad<\frac{1}{4}
\end{aligned}
$$

Now using (13) and (16) the squeezing conditions in (18) can be reduced to the following forms:

$$
\begin{aligned}
& F_{1}=<a^{\dagger^{2}}>+<a^{\dagger} a>-2<a^{\dagger}>^{2}=\beta^{2} I_{2}+I_{3}-2 \beta^{2} I_{1}^{2}<0 \\
& \text { or } \\
& G_{1}=<a^{\dagger} a>-<a^{\dagger}>^{2}=I_{3}-\beta^{2} I_{2}<0
\end{aligned}
$$

where $\beta$ is taken to be real and $I_{i}, i=1,2,3$ are infinite series whose explicit forms are given in the appendix.

We now evaluate the inequalities in (19) and the results are presented in fig 1 . In fig 1 we have plotted graphs of $F_{1}$ and $G_{1}$ against $\beta$ for fixed $\eta$. From fig 1 it is seen that while the curve of $F_{1}$ is greater than zero that of $G_{1}$ is less than zero for a wide range of $\beta$. Thus one of the inequalities in (19) is satisfied. This implies that the nonlinear coherent state exhibits quadrature squeezing. We would like to mention here that we have examined the inequalities in (18) for a wide range of values of $\beta$ and $\eta$ and obtained the same qualitative behaviour as in fig 1.

\subsection{Amplitude squared squeezing}

In order to examine whether or not the nonlinear coherent state exhibits amplitude squared squeezing we introduce the following hermitian operators:

$$
X_{2}=\frac{\left(a^{2}+a^{\dagger^{2}}\right)}{2}, Y_{2}=\frac{\left(a^{2}-a^{\dagger^{2}}\right)}{2 i}
$$

Then $X_{2}$ and $Y_{2}$ obey the uncertainty relation

$$
<\Delta X_{2}^{2}>\quad<\Delta Y_{2}^{2}>\geq \frac{1}{4}\left|<\left[X_{2}, Y_{2}\right]>\right|^{2}
$$


From (21) it follows that the nonlinear coherent state will exhibit amplitude squared squeezing if

$$
\begin{aligned}
& <\Delta X_{2}^{2}>\quad<\frac{1}{2}\left|<\left[X_{2}, Y_{2}\right]>\right| \\
& \text { or } \\
& <\Delta Y_{2}^{2}>\quad<\frac{1}{2}\left|<\left[X_{2}, Y_{2}\right]>\right|
\end{aligned}
$$

Now proceeding as before the conditions (22) for amplitude squared squeezing become

$$
\begin{aligned}
& F_{2}=<a^{\dagger^{4}}>+<a^{\dagger^{2}} a^{2}>-<a^{\dagger^{2}}>=\beta^{4} I_{4}+I_{5}-I_{2}^{2}<0 \\
& \text { or } \\
& G_{2}=<a^{\dagger^{4}}>-<a^{\dagger^{2}} a^{2}>=I_{5}-\beta^{4} I_{4}<0
\end{aligned}
$$

where $I_{4}$ and $I_{5}$ are infinite series whose exact forms are given in the appendix. To examine the inequalities in (23) we plot $F_{2}$ and $G_{2}$ against $\beta$ for $\eta$ fixed. From figure 2 we find that $F_{2}<0$ for a certain range of $\beta$ and subsequently it becomes positive. On the other hand $G_{2}$ is always positive. This implies that the nonlinear coherent state $\mid \beta>$

exhibits amplitude squared squeezing in the $X_{2}$ component. As in the case of quadrature squeezing we have examined the inequalities in (23) for different values of $\beta$ and $\eta$ and it has been found that although squeezing can be increased (or decreased) by changing the parameter values the basic qualitative features remain the same as in fig 2 .

\subsection{Sub-Poissonian Behaviour}

To examine sub-Poissonian behaviour we consider the second order correlation function $g^{2}(0)$ defined by

$$
g^{2}(0)=\frac{<a^{\dagger^{2}} a^{2}>}{<a^{\dagger} a>^{2}}=\frac{I_{4}}{I_{3}^{2}}
$$

Then the state exhibits super-Poissonian/Poissonian/sub-Poissonian behaviour according to

$$
\begin{aligned}
g^{2}(0) & >1 \\
& <
\end{aligned}
$$

We now plot $g^{2}(0)$ against $\beta$ keeping $\eta$ constant. From fig 3 it is seen that $g^{2}(0)<1$ for the range of $\beta$ considered. This implies that the nonlinear coherent state $\mid \beta>$ exhibits sub-Poissonian behaviour. It may be noted that $g^{(2)(0)}$ has an increasing trend and so for sufficiently large values of $\beta$ it may show super-Poissonian behaviour. 


\section{Conclusion}

In this paper we have used a displacement type operator to construct a new class of nonlinear coherent states which are distinct from those which are annihilation operator eigenstates [3, [4. It has been shown that this nonlinear coherent state exhibits interesting nonclassical properties like squeezing and sub-Poissonian behaviour. We feel it would be interesting to examine other properties e.g., quantum interference ,phase properties etc of the nonlinear coherent state $\mid \beta>$.

\section{Appendix}

In order to examine squeezing and antibunching we need to evaluate several expectation values like $<a^{\dagger}>,<a^{\dagger^{2}}>,<a^{\dagger} a>$ etc. These expectation values are given in terms of the following series:

$$
\begin{gathered}
\beta^{-1}<a>=\beta^{*^{-1}}<a^{\dagger}>=I_{1}=c^{2} \sum_{n=0}^{\infty} \frac{\left(\beta^{*} \beta\right)^{n} f(n) ! f(n+1) !}{n !} \\
\beta^{-2}<a^{2}>=\beta^{*^{-2}}<a^{\dagger^{2}}>=I_{2}=c^{2} \sum_{n=0}^{\infty} \frac{\left(\beta^{*} \beta\right)^{n} f(n) ! f(n+2) !}{n !} \\
<a^{\dagger} a>=I_{3}=c^{2} \sum_{n=0}^{\infty} \frac{\left(\beta^{*} \beta\right)^{n+1}[f(n+1) !]^{2}}{n !} \\
<a^{\dagger^{2}} a^{2}>=I_{4}=c^{2} \sum_{n=0}^{\infty} \frac{\left(\beta^{*} \beta\right)^{n}[f(n+2) !]^{2}}{n !} \\
\beta^{-4}<a^{4}>=\beta^{*^{-4}}<a^{\dagger^{4}}>=I_{5}=c^{2} \sum_{n=0}^{\infty} \frac{\left(\beta^{*} \beta\right)^{n} f(n) ! f(n+4) !}{n !}
\end{gathered}
$$




\section{References}

[1] A.M.Perelemov, Generalsied coherent states and their applications (SpringerVerlag,Berlin, 1996)

[2] W.M.Zhang, Rev.Mod.Phys 62, 867 (1990)

[3] R.L.de Matos Filho and W.Vogel, Phys.Rev A54, 4560 (1996)

[4] V.I.Manko,G.Marmo,E.C.G.Sudarshan and F.Zaccaria, Phys.Scrip 55,528 (1997)

[5] Ya.I.Granovoskii, I.M.Lutzenko and A.S.Zhedanov, Ann.Phys 217, 1 (1992)

[6] L.Vinet and P.Letourneau, Ann.Phys 243, 144 (1995)

[7] G.Junker and P.Roy, Phys.Lett A232,155 (1997);ibid A257, 113 (1999)

[8] V.P.Karassiov, J.Phys A27, 153 (1994)

[9] C.D.J.Fernandez, V.Hussin and L.M.Nieto, J.Phys A27,3547 (1994)

[10] C.D.J.Fernandez, L.M.Nieto and O.Rosas-Ortiz, J.Phys A28, 2693 (1995)

[11] V.Sunilkumar, B.Bambah, P.Panigrahi and V.Srinivasan, preprint quant-ph/9905010

[12] V.Sunilkumar, B.A.Bambah, R.Jagannathan, P.K.Panigrahi and V.Srinivasan, preprint quant-ph/9907075

[13] W.H.Louisell, Quantum Statistical Properties of Radiation, J.Willey,New York 1973.

[14] K.Fuji and T.Suzuki, preprint hep-th/9907084

[15] B.Roy and P.Roy, J.Opt B1, 341 (1999) 


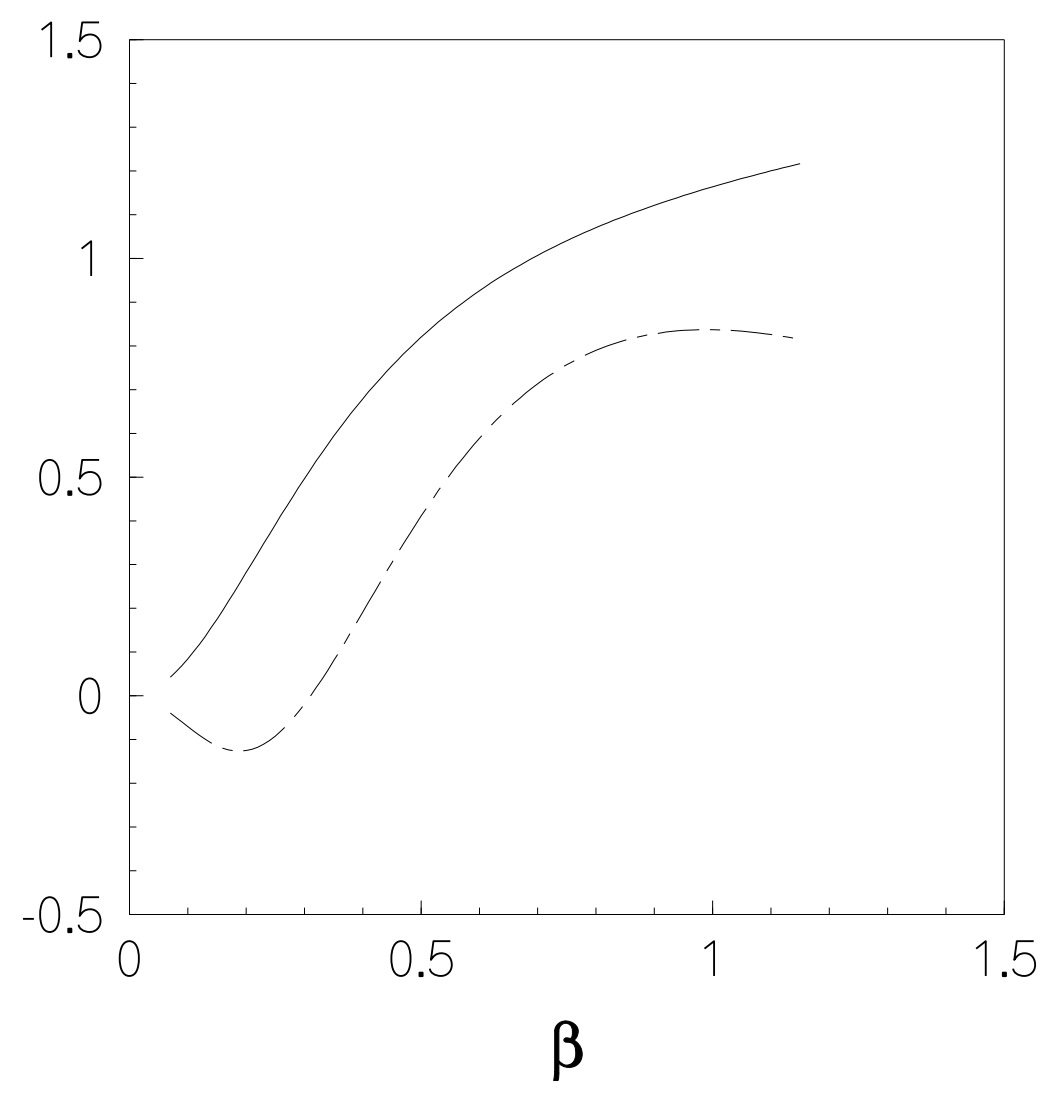

Fig 1. Quadrature squeezing of nonlinear coherent state for $\boldsymbol{\eta}=.7$. Solid curve $\left(F_{1}\right)$ and broken curve $\left(G_{1}\right)$ 


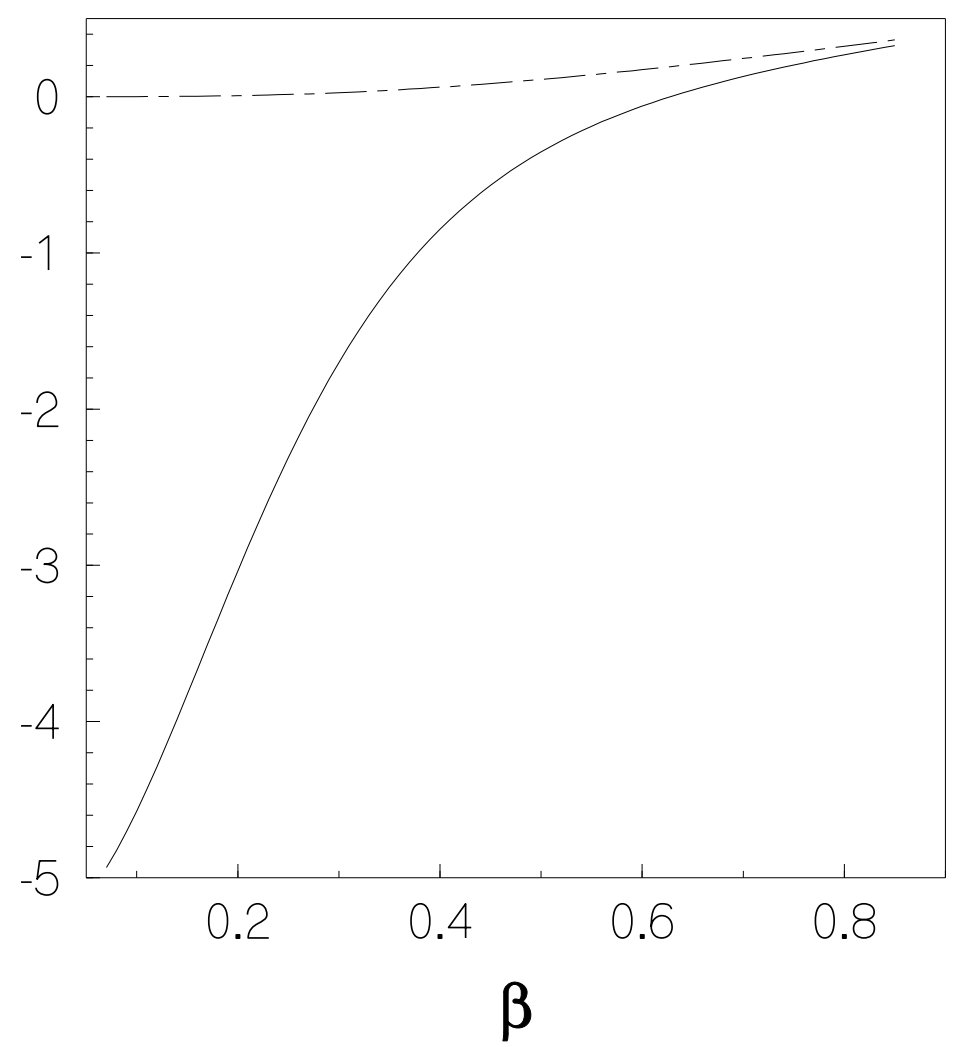

Fig 2. Amplitude squared squeezing of nonlinear coherent state for for $\boldsymbol{\eta}=$.8. Solid curve $\left(F_{2}\right)$ and broken curve $\left(G_{2}\right)$ 


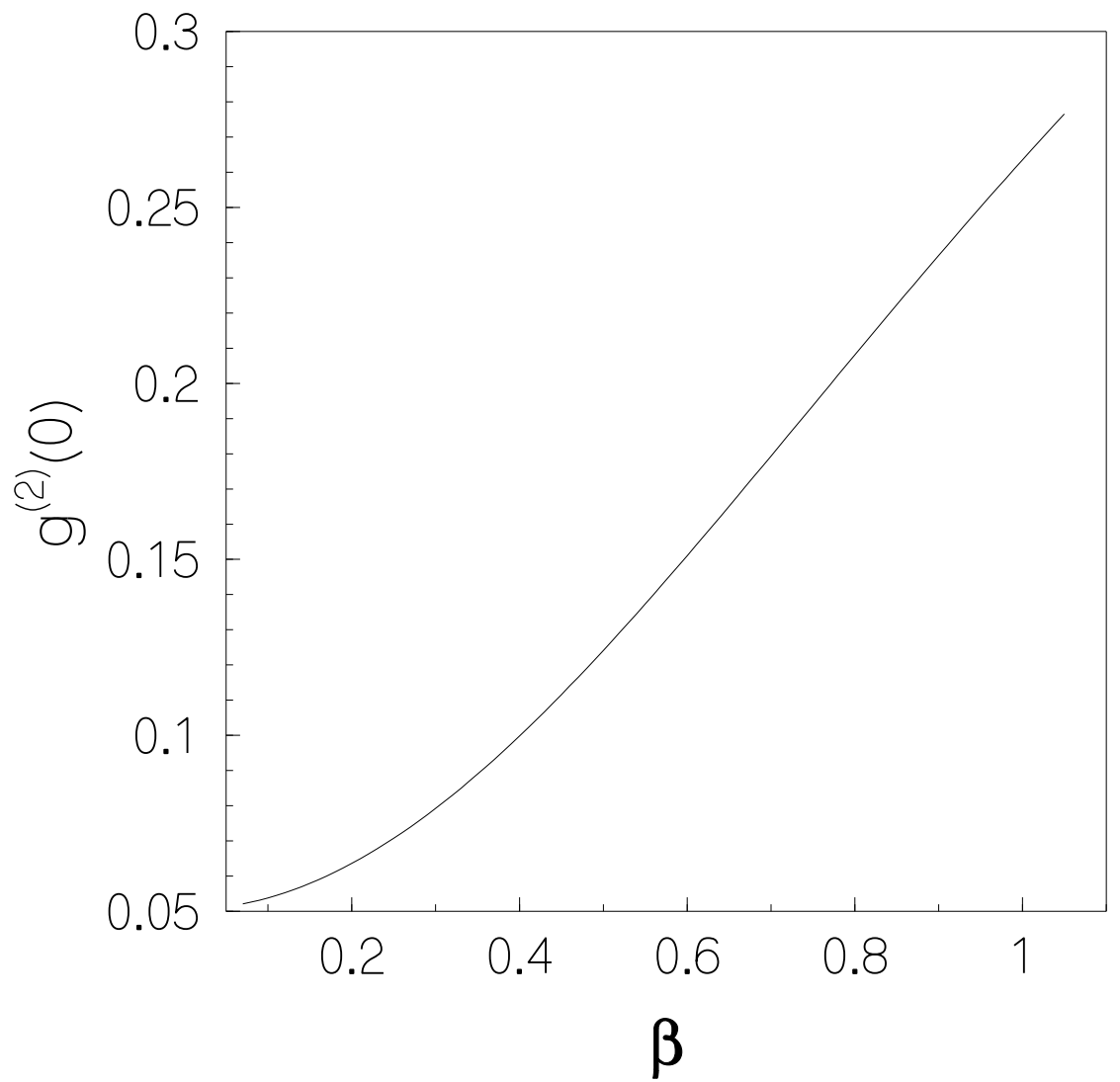

Fig 3.Sub-Poissonian behaviour of nonlinear coherent state for $\eta=.7$ 